\title{
Supermodular Functions on Finite Lattices
}

\author{
S. DAVID PROMISLOW ${ }^{1}$ and VIRGINIA R. YOUNG ${ }^{2, *}$ \\ ${ }^{1}$ Department of Mathematics and Statistics, York University, Toronto, ON M3J 1P3, Canada. \\ e-mail: promis@yorku.ca \\ ${ }^{2}$ Department of Mathematics, University of Michigan, Ann Arbor, MI 48109, USA. \\ e-mail:vryoung@umich.edu
}

(Received: 2 August 2005; in final form: 21 October 2005)

\begin{abstract}
The supermodular order on multivariate distributions has many applications in financial and actuarial mathematics. In the particular case of finite, discrete distributions, we generalize the order to distributions on finite lattices. In this setting, we focus on the generating cone of supermodular functions because the extreme rays of that cone (modulo the modular functions) can be used as test functions to determine whether two random variables are ordered under the supermodular order. We completely determine the extreme supermodular functions in some special cases.
\end{abstract}

Mathematics Subject Classification (2000): 06B99 (Primary), 52A20 (Secondary).

Key Words: convex cones, extreme rays, supermodular functions, ordering random variables.

\section{Introduction}

Traditionally, the supermodular order on multivariate distributions has been used to compare the riskiness of multivariate random variables on $\mathcal{I}^{k}$, in which $\mathcal{I}$ is a totally-ordered set, such as the real numbers, the positive integers, or some subset of these sets, and $k$ is a positive integer. Note that $\mathcal{I}^{k}$ is a lattice under the partial order given by $\mathbf{x} \leq \mathbf{y}$ if each coordinate of $\mathbf{x} \in \mathcal{I}^{k}$ is less than or equal to the corresponding coordinate of $\mathbf{y} \in \mathcal{I}^{k}$. In fact, one can consider real-valued random variables defined on lattices $L$ more general than $\mathcal{I}^{k}$, and we do so in this paper. Recall that a lattice is a set equipped with a partial order such that every pair of elements $(x, y)$ has a least upper bound $x \vee y$ and a greatest lower bound $x \wedge y$.

To define the supermodular order on random variables on lattices, we begin by saying that a real-valued function defined on a lattice, $f: L \rightarrow \mathcal{R}$, is supermodular if

$$
f(x \vee y)+f(x \wedge y) \geq f(x)+f(y),
$$

\footnotetext{
* Corresponding author.
} 
for all $x, y \in L$. Next, one says that a random variable $X$ on $L$, endowed with some probability measure, is dominated by $Y$ in supermodular order if

$$
E[f(X)] \leq E[f(Y)],
$$

for all real-valued supermodular functions $f$ on $L$ for which the expectations in (1.2) exist. See Shaked and Shanthikumar [11], Müller and Scarsini [8], Müller and Stoyan ([9]; Section 3.9), and Promislow [10] for more on the supermodular order.

In this paper, we consider the problem of determining when two random variables are related in the supermodular order. Our work is inspired by the following example.

EXAMPLE 1.1. Two real-valued random variables on $\mathcal{R}$, endowed with a probability measure, are said to be ordered via first-stochastic dominance if inequality (1.2) holds for all real-valued increasing functions $f$ on $\mathcal{R}$, for which the expectations exist. To apply this definition to determine whether two random variables are so ordered can be difficult. However, there is a subset of increasing functions for which it is easy to check (1.2). Indeed, consider the collection of increasing step functions $f_{a}$ of the form $f_{a}(x)=1$ if $x>a$ and $f_{a}(x)=0$ otherwise, for an arbitrary real number $a \in \mathcal{R}$. Then, (1.2) becomes

$$
\operatorname{Pr}(X>a) \leq \operatorname{Pr}(Y>a) .
$$

It is well known that if (1.3) holds for all real numbers $a$, then $Y$ dominates $X$ in first-stochastic dominance order; that is, (1.2) holds for all increasing functions, subject to some technical requirements. See, for example, Mosler and Scarsini [6] and the references therein. For more recent references on this topic, see Müller [7] and Müller and Stoyan ([9]; Section 2.5). Note that (1.3) is certainly easier to verify for all a than is (1.2) for all increasing functions $f$.

Similarly, it can be difficult to check whether two random variables are ordered in the supermodular order. Promislow [10] shows that the supermodular order is equivalent to one involving a sequence of mass shifts, for which dominance might be easier to determine. In this paper, we take the approach in Example 1.1 by seeking a representative subset (or test set) of supermodular functions such that if (1.2) holds for all the functions in the test set, then it holds for all supermodular functions.

In the next section, we present our basic framework and introduce the idea of extreme supermodular functions on lattices. It is the set of extreme supermodular functions that (in some sense) will serve as our test set. In Section 3, we find the extreme supermodular functions on chain lattices, that is, lattices formed by joining disjoint chains at their maximum and minimum. In Section 4, we turn our attention to the lattices $Z_{n}^{k}$ and closely examine the lattice $Z_{2}^{k}$, the lattice of subsets of a $k$-element set. In Section 5, we determine the extreme supermodular 
functions on $Z_{2}^{4}$. In Section 6 , we focus on $Z_{n}^{3}$ and determine the extreme supermodular functions on $Z_{3}^{3}$. Section 7 concludes the paper.

\section{Basic Definitions}

Let $L$ be a finite lattice with $d$ elements, and let 1 and 0 denote the maximum and minimum elements of $L$, respectively. We denote the space of all real-valued functions on $L$ by $\mathcal{F}$. For each $f \in \mathcal{F}$ and for each pair of elements $(x, y) \in$ $L \times L$, we define

$$
\delta_{f}(x, y)=f(x \vee y)+f(x \wedge y)-f(x)-f(y) .
$$

Note that if $x$ and $y$ are comparable, then $\delta_{f}(x, y)=0$.

We say that the pair $(x, y)$ is $f$-modular if $\delta_{f}(x, y)=0$. We say that the function $f$ is modular if every incomparable pair is $f$-modular. From (1.1), note that function $f$ is supermodular if $\delta_{f}(x, y) \geq 0$ for all $x, y \in L$.

Denote the convex cone of supermodular functions by $\mathcal{S}$ and the subspace of modular functions by $\mathcal{M}$. As an aside, note that the supermodular functions $\mathcal{S}$ do form a cone in $\mathcal{R}^{d}$. Recall that a cone in a vector space is a set $\mathcal{K}$ such that $0 \in \mathcal{K}$ and $\lambda x \in \mathcal{K}$ for every $\lambda \geq 0$ and every $x \in \mathcal{K}$. Moreover, $\mathcal{S}$ is convex: Let $0 \leq$ $\beta \leq 1$ and let $f, g \in \mathcal{S}$; then, $\beta f+(1-\beta) g \in \mathcal{S}$ because $\delta_{\beta f+(1-\beta) g}=\beta \delta_{f}+$ $(1-\beta) \delta_{g}$. Thus, the supermodular order is generated by a cone of functions, as is the first-stochastic dominance order; see Marshall [5], Whitt [13], and Müller and Stoyan (9; Chapter 2) for work on orders generated by cones of functions.

Our goal is to find a set of supermodular functions, or generators, $\mathcal{E}=$ $\left\{e_{1}, e_{2}, \ldots, e_{m}\right\}$ such that every supermodular function $f$ can be written as $f=$ $\sum_{i=1}^{m} \alpha_{i} e_{i}$ for $\alpha_{i} \geq 0, i=1,2, \ldots, m$. We know that such a set exists, since $\mathcal{S}$ is the intersection of finitely many closed halfspaces; see Ziegler (14, Theorem 1.3). In particular, $\mathcal{S}$ is the intersection of $H_{x, y}=\left\{f \in \mathcal{F}: \delta_{f}(x, y) \geq 0\right\}$ as $x, y$ runs over the incomparable pairs of $L$. It is usually not difficult to find a basis for the subspace $\mathcal{M}$, which means we can restate our goal with the quotient $\mathcal{S} / \mathcal{M}$ in place of $\mathcal{S}$. We can then check that $X$ is dominated by $Y$ in the supermodular order by verifying that inequality (1.2) holds for each generator in $\mathcal{S} / \mathcal{M}$ and that (1.2) holds with equality for each basis element in $\mathcal{M}$. See Müller and Stoyan (9; Theorem 2.5.1) for a related result.

The advantage of moving to the quotient cone is that $\mathcal{S} / \mathcal{M}$ is a convex cone without lines, and we can show that a minimal generating set $\mathcal{E}$ of $\mathcal{S} / \mathcal{M}$ must be precisely generators of the extreme rays of this cone. We will review briefly the relevant facts. The nonnegative multiples of an element in a cone $K$ form a ray. We say that the ray $K_{1} \subset K$ is extreme if for any $a \in K_{1}$ and any $b, c \in K$ such that $a=b+c$, then we have $b, c \in K_{1}$. A set $B$ is called a base of $K$ if $0 \notin B$ and for every point $a \in K$ such that $a \neq 0$, there is a unique representation $a=\alpha b$ with $b \in B$ and $\alpha>0$. If $K$ has a compact, convex base then it follows from a version of the Krein-Milman Theorem [1] that every point $a \in K$ can be written 
as $\sum_{i=1}^{m} \alpha_{i} e_{i}$ for $\alpha_{i} \geq 0$, in which the points $e_{i}$ span the extreme rays of $K$. In contrast, it is of interest to note that a cone containing a nonzero subspace $M$ has no extreme rays since for any nonzero $a \notin M$ and nonzero $m \in M$, $a=(a-m)+m$ and $m$ is not in the ray generated by $a$, while for nonzero $a \in M, a=2 a+(-a)$ and $-a$ is not in the ray generated by $a$.

To verify that $\mathcal{S} / \mathcal{M}$ has a compact, convex base, we define the linear functional $\mathcal{L}$ on $\mathcal{F} / \mathcal{M}$ by $\mathcal{L}(f)=\sum_{x, y} \delta_{f}(x, y)$, and note that $\mathcal{L}$ is positive on the nonzero elements of $\mathcal{S} / \mathcal{M}$. For our base, we take $\{k \in \mathcal{S} / \mathcal{M}: \mathcal{L}(k)=1\}$. This is clearly a base, convex and closed. It is bounded due to the following lemma, which is probably well known, but for lack of a suitable reference, we include a proof.

LEMMA 2.1. Let $K$ be a closed cone in $\mathcal{R}^{d}$ and let $\mathcal{L}$ be a linear functional on $K$ such that $\mathcal{L}(k)>0$ for all $k \neq 0 \in K$. Then, $\{k \in K: \mathcal{L}(k)=1\}$ is bounded under the usual metric $\|\cdot\|$ in $\mathcal{R}^{d}$.

Proof. Suppose not; then, for any integer $n$, we can find $k_{n} \in K$ such that $\mathcal{L}\left(k_{n}\right)=1$ and $\left\|k_{n}\right\| \geq n$. Let $x_{n}=k_{n} /\left\|k_{n}\right\|$; then, $0<\mathcal{L}\left(x_{n}\right) \leq 1 / n$, and $\left\|x_{n}\right\|=1$. By compactness of the unit sphere, we can suppose that $x_{n} \rightarrow x$ for some $x$ with $\|x\|=1$, so $x \neq 0$. Since $K$ is closed, $x \in K$. But $\mathcal{L}(x)=\lim _{n \rightarrow \infty}$ $\mathcal{L}\left(x_{n}\right)=0$, a contradiction to the hypothesis.

A similar problem arises in polymatroid theory where the object of interest is the collection of submodular functions, that is, those satisfying (1.1) with the inequality reversed. Edmonds [2] proposed the problem of finding the extreme rays of the cone of functions on $Z_{2}^{k}$ that are submodular, increasing, and assign 0 to 0 . It can be shown that this cone is isomorphic to a direct sum of $\mathcal{S} / \mathcal{M}$ and a certain cone of modular functions (specifically, the positive measures - see the remark at the end of Section 4.1), so for the lattice $Z_{2}^{k}$, the two problems are essentially equivalent. We find the cone $\mathcal{S} / \mathcal{M}$ easier to deal with and more natural for our particular application. Kovalëv [3] and Kovalëv et al. [4] investigate extreme rays for certain subcones of submodular functions.

The rays of the cone $\mathcal{S} / \mathcal{M}$ can be identified with the set $\mathcal{Q}$ of equivalence classes under the equivalence relation $\sim$ on $\mathcal{S}$, whereby $f \sim g$ if $f-\alpha g \in \mathcal{M}$ for some $\alpha>0$. We let $[f]$ denote the equivalence class of $f$. We will, in what follows, refer to a function $f \in \mathcal{S}$ as being extreme to mean that $[f]$ is an extreme ray in $\mathcal{Q}$. It follows that a function $f \in \mathcal{S}$ is extreme, according to this definition, if and only if $f=g+h$, for $g, h \in \mathcal{S}$, implies that either $g \in \mathcal{M}$ or $g \sim f$.

For $f, g \in \mathcal{S}$, we say $g \preceq f$ if all $f$-modular pairs are also $g$-modular. This defines a relation on $\mathcal{Q},[g] \preceq[f]$, which is well-defined, since if $(x, y)$ is $f$ modular, then it is $g$-modular for all $g \sim f$. This relation is clearly transitive and reflexive. It is not an order, but rather a preorder, because we can have two distinct elements with precisely the same $f$-modular pairs. For example, for any $f$ that takes zero values except for positive values on 1 and 0 , the $f$-modular pairs 
are all those $(x, y)$ for which neither $x \vee y=1$ nor $x \wedge y=0$. The importance of this pre-order is given by the following.

LEMMA 2.2. If $f=g+h$, in which $f, g, h \in \mathcal{S}$, then $g \preceq f$.

Proof. Note that from (2.1),

$$
\delta_{f}(x, y)=\delta_{g}(x, y)+\delta_{h}(x, y) \geq \delta_{g}(x, y) \geq 0 .
$$

Therefore, if $(x, y)$ is $f$-modular, then $\delta_{f}(x, y)=0$, from which it follows that $\delta_{g}(x, y)=0$, or equivalently, $(x, y)$ is $g$-modular.

This lemma leads to an equivalent characterization of extreme functions.

THEOREM 2.3. $[f]$ is extreme if and only if $[f]$ is a minimal non-modular element with respect to $\preceq$. That is, for $g \notin \mathcal{M},[g] \preceq[f]$ implies that $g \sim f$.

Proof. Suppose that $[f]$ is minimal non-modular with respect to $\preceq$ and that $f=g+h$, in which $g, h \in \mathcal{S}$ and $g \notin \mathcal{M}$. Then, by Lemma 2.2, we have $g \preceq f$ and by minimality, we must have $g \sim f$.

Conversely, suppose that $[f]$ is not minimal non-modular. Then, we can find $g$ in $\mathcal{S}$ but not in $\mathcal{M}$, for which $g \not f$ and $g \preceq f$. Any pair $(x, y)$ that is not $g$ modular is also not $f$-modular, so we can choose a positive $\epsilon$ that is less than $\delta_{f}(x, y) / \delta_{g}(x, y)$ for all non $g$-modular pairs $(x, y)$. Then, $f-\epsilon g \in \mathcal{S}$, and the decomposition $f=(f-\epsilon g)+\epsilon g$ shows that $f$ is not extreme.

We will often proceed in the following manner. We are given a set $\mathcal{E}$ of extreme (supermodular) functions on a given lattice $L$, and we want to show that these constitute, up to equivalence, all the extreme functions. There are two ways to proceed. One is to show that any function $f \in \mathcal{S}$ can be written as positive linear combination of functions in $\mathcal{E}$. Another is to show that for any $f \in \mathcal{S}$ there is an $e \in \mathcal{E}$ such that $e \preceq f$. If we have identified $\mathcal{E}$ as a complete set of extreme functions by this latter method, it will turn out that any $f \in \mathcal{S}$ can be written as a positive linear combination of functions in $\mathcal{E}$, by invoking Theorem 2.3 and the Krein-Milman Theorem [1].

The following definitions and examples introduce supermodular functions that we use later in finding extreme functions on various lattices.

DEFINITION 2.4. We say that $a \in L$ is indecomposable if there does not exist an incomparable pair $(x, y)$ such that $x \vee y=a$ and there does not exist an incomparable pair $(v, w)$ such that $v \wedge w=a$. We say that $a \in L$ is quasiindecomposable if $x \vee y=a$ implies that $x \wedge y=0$ and if $x \wedge y=a$ implies that $x \vee y=1$, where in either case, $(x, y)$ is an incomparable pair. For an indecomposable $a$, let $i n d_{a}$ denote the function that takes the value of -1 at $a$ and zero elsewhere. For a quasi-indecomposable $a$, let $q i n_{a}$ denote the function 
that takes the value of -1 at $a$, the value of 1 at 0 and at 1 , and the value of 0 elsewhere. It is clear that these functions are supermodular.

EXAMPLE 2.5. In the lattice of subsets of a two-element set, the singletons are indecomposable. In the lattice of subsets of a four-element set, the two-element sets are quasi-indecomposable.

DEFINITION 2.6. Two common supermodular functions on a lattice are (1) the upper function $u_{x}$ for $x \in L$ that takes the value 1 on all points greater than or equal to $x$ and the value of zero elsewhere and (2) the lower function $\ell_{x}$ that takes the value 1 on all points less than or equal to $x$ and the value 0 elsewhere. In Definition 2.8 below, we introduce a generalization of these functions that involve the concept of fans.

An upper fan $F$ in $L$ is a subset of pairwise incomparable points $\left(a_{1}\right.$, $\left.a_{2}, \ldots, a_{m}\right)$, for which each pair of distinct points $\left(a_{i}, a_{j}\right)$ has the same least upper bound. We call the index $m$ the length of the fan. A lower fan $G$ in $L$ is a subset of incomparable points $\left(a_{1}, a_{2}, \ldots, a_{m}\right)$, for which each pair of distinct points $\left(a_{i}, a_{j}\right)$ has the same greatest lower bound; that is, $G$ is an upper fan in the dual lattice.

EXAMPLE 2.7. In the lattice of all subsets of a four-element set $\{a, b, c, d\}$, we can list all upper fans. In this example and in Sections 4 and 5, we write, say, $a b$ to denote $\{a, b\}$. For example, $\{a b, a c d, b c d\}$ should more properly be written as $\{\{a, b\},\{a, c, d\},\{b, c, d\}\}$, but we find the latter notation cumbersome. The upper fans of this lattice are as follows:

(i) Any singleton in the lattice, that is, any subset of $\{a, b, c, d\}$ (the restriction is vacuous);

(ii) Any doubleton of incomparable points in the lattice, that is, any two subsets of $\{a, b, c, d\}$ neither of which is a subset of the other;

(iii) $\{a b, a c, b c\}$;

(iv) $\{a b, a c d, b c d\}$;

(v) Any three of the three-element subsets; and

(vi) All four of the three-element subsets;

where in (iii) and (iv), we include all sets arising from the given one by a permutation of the elements in $\{a, b, c, d\}$.

DEFINITION 2.8. Given an upper fan $F$ (see Definition 2.6), for which $b$ is the common least upper bound of any pair of points, let $u_{F}$ denote the function that takes the value 1 on all points $x$ such that for some $i, a_{i} \leq x$, but $b \leq x x$; the value 2 on all $x$ such that $b \leq x$; and the value 0 elsewhere. Note that $u_{F}$ generalizes the notion of upper function $u_{x}$.

For a lower fan $G$, for which $c$ is the common greatest lower bound of any pair of points, let $\ell_{G}$ denote the functions that takes the value 1 on all points $x$ 
such that for some $i, x \leq a_{i}$ but $x \not \leq c$; the value 2 on all $x$ such that $x \leq c$; and the value 0 elsewhere. Note that $\ell_{G}$ generalizes the notion of lower function $\ell_{x}$.

\section{THEOREM 2.9. The functions $u_{F}$ and $\ell_{G}$ are supermodular.}

Proof. We prove this for $u_{F}$. We consider various cases for incomparable pairs $(x, y)$ and show that $f(x \vee y)+f(x \wedge y) \geq f(x)+f(y)$ in each case.

- If $u_{F}(x)=1$ and $u_{F}(y)=0$, then for some $i$, we have $a_{i} \leq x$, which implies that $a_{i} \leq x \vee y$. Thus, $u_{F}(x \vee y) \geq 1$.

- If $u_{F}(x)=1$ and $u_{F}(y)=1$, then for some $i$ and $j$, we have $a_{i} \leq x$ and $a_{j} \leq y$. If $i=j$, then $u_{F}(x \vee y) \geq 1$ and $u_{F}(x \wedge y) \geq 1$. If $i \neq j$, then $b \leq x \vee y$, so that $u_{F}(x \vee y)=2$.

- If $u_{F}(x)=2$ and $u_{F}(y)=0$, then $u_{F}(x \vee y)=2$.

- If $u_{F}(x)=2$ and $u_{F}(y)=1$, then for some $i$, we have $a_{i} \leq y \leq b$ and $a_{i} \leq b \leq x$, so that $a_{i} \leq x \wedge y$. Hence, $u_{F}(x \wedge y)=1$, and $u_{F}(x \vee y)=2$.

- If $u_{F}(x)=2$ and $u_{F}(y)=2$, then $u_{F}(x \vee y)=u_{F}(x \wedge y)=2$.

\section{Extreme Functions on Chain Lattices}

In this section, we find a basis for the modular functions and a complete set of extreme supermodular functions on any chain lattice. A chain lattice is a lattice that consists of the joining of disjoint chains at their maximum and minimum.

Specifically, let $C\left(m_{1}, m_{2}, \ldots, m_{k}\right)$ denote the lattice that consists of the points 0,1 , and $x_{i j}$, in which $j=1,2, \ldots, m_{i}$ and $i=1,2, \ldots, k$. The order is given by $0 \leq x_{i j} \leq 1$ for all $i, j ; x_{i j} \leq x_{i l}$ if and only if $j \leq l$; and for $i \neq h, x_{i j}$ and $x_{h l}$ are incomparable for all $j, l$. In other words, the lattice consists of $k$ chains that are disjoint except for 0 and 1 . We let $C_{i}$ denote the set $\left\{0,1, x_{i j}\right.$ for $j=1$, $\left.2, \ldots, m_{i}\right\}$. The points other than 0 or 1 will be called the intermediate points of $C_{i}$.

We first determine the subspace of modular functions $\mathcal{M}$ on $C\left(m_{1}\right.$, $\left.m_{2}, \ldots, m_{k}\right)$. For $k>2, \mathcal{M}$ is 2-dimensional, consisting of the functions $M(a, b)$ that take the value of $a$ at $1, b$ at 0 , and $(a+b) / 2$ on all intermediate points. For $k=2, \mathcal{M}$ is 3-dimensional, consisting of the functions $M(a, b, c)$ that take the value of $a$ at $1, b$ at $0, c$ at all intermediate points of $C_{1}$, and $a+b-c$ on all intermediate points of $C_{2}$. We say that a function on a chain lattice is in normal form if it takes the value 0 at both 1 and 0 . If $k>2$, it is clear that for each $f \in \mathcal{S}$, there is a $g \in \mathcal{S}$ in normal form such that $f \sim g$, in which $g$ is unique up to positive scalar multiple.

As a first step in seeking extreme supermodular functions, we have the following lemma.

LEMMA 3.1. Suppose $k>2$. Then, for all $i=1,2, \ldots, k$, the upper function $u_{x_{i 1}}$ is extreme. 
Proof. We can take as normal form for $u_{x_{i 1}}$ a function that takes the value 1 on the intermediate points of $C_{i}$ and a value of -1 on all other intermediate points. Take any $f$ in normal form such that $f \preceq u_{x_{i 1}}$. Suppose that $f\left(x_{i 1}\right)=\alpha$. Then, for all $h \neq i$ and for all $l=1,2, \ldots, m_{h}$, we have that $\left(x_{i 1}, x_{h l}\right)$ is $u_{x_{i 1}}$ modular and, therefore, $f$-modular. Hence, $\alpha+f\left(x_{h l}\right)=0$, or $f\left(x_{h l}\right)=-\alpha$. We similarly conclude that $f\left(x_{i j}\right)=\alpha$ for all $j=1,2, \ldots, m_{i}$, thereby showing that $f$ is equivalent to a nonnegative multiple of $u_{x_{i 1}}$. Note that we must have $\alpha \geq 0$, since if not, we could contradict the supermodularity of $f$ by looking at intermediate points on chains $C_{h}$ and $C_{j}$, where $h$ and $j$ are distinct indices and neither is equal to $i$.

Remark. For $k=2$ and $i=1,2$, the functions $u_{x_{i 1}}$ are easily seen to be modular.

In the lattice $C\left(m_{1}, m_{2}, \ldots, m_{k}\right)$, all the intermediate points are indecomposable. Let $s_{i j}$ denote the supermodular function $i n d_{x_{i j}}$; that is, $s_{i j}$ takes the value -1 on $x_{i j}$ and 0 elsewhere. It is natural to ask when this function is extreme.

\section{THEOREM 3.2.}

(a) If $k>3$, then $s_{i j}$ is extreme for all $j=1,2, \ldots, m_{i}$ and $i=1,2, \ldots, k$.

(b) Suppose $k=3$. If $m_{i}>1$, then $s_{i j}$ is extreme for $j=1,2, \ldots, m_{i}$. If $m_{i}=1$, then $s_{i 1} \sim \sum_{j \neq i} u_{x_{j 1}}$.

(c) Suppose $k=2$. If $m_{i}>1$, then $s_{i j}$ is extreme for $j=1,2, \ldots, m_{i}$. If, say $m_{1}=1$, then $s_{11} \sim \sum_{j=1}^{m_{2}} s_{2 j}$.

\section{Proof.}

(a) We will show that for $f \in \mathcal{S}$ in normal form with $f \preceq s_{i j}$, it follows that $f$ is a nonnegative multiple of $s_{i j}$. The modular pairs for $s_{i j}$ are those that do not include the point $x_{i j}$. Take any two points other than $x_{i j}$ that are in different chains. The sum of the values of $f$ on these two points must then equal 0 . Since we have at least three such chains from which to choose, the only way this can occur is if $f$ takes the value 0 on all such points. Because $f$ is supermodular, $f\left(x_{i j}\right) \leq 0$. Thus, $f$ equals a nonnegative multiple of $s_{i j}$.

(b) When $k=3$, then we still have three chains from which to choose, except when $m_{i}=1$. For the final statement, note that when $m_{i}=1$,

$$
s_{i 1}+M(2,0)=\sum_{j \neq i} u_{x_{j 1}} .
$$

(c) Suppose $m_{1}>1$; then, take $f \preceq s_{1 j}$ in normal form. It follows that for any $h \neq j$ and for all $l=1,2, \ldots, m_{2}$, we have $f\left(x_{1 h}\right)=-f\left(x_{2 l}\right)$. By adding to $f$ 
the modular function $M\left(0,0,-f\left(x_{1 h}\right)\right)$, we obtain that $f$ is equivalent to a nonnegative multiple of $s_{1 j}$. For the final statement, note that when $m_{1}=1$,

$$
s_{11}+M(0,0,1)=\sum_{h=1}^{m_{2}} s_{2 h} .
$$

THEOREM 3.3. A complete set of extreme supermodular functions for the chain lattice $C\left(m_{1}, m_{2}, \ldots, m_{k}\right)$ is given as follows:

(a) When $k>3,\left\{s_{i j}, u_{x_{i 1}}: j=1,2, \ldots, m_{i}, i=1,2, \ldots, k\right\}$ is a complete set of extreme functions.

(b) When $k=3,\left\{s_{i j}: m_{i}>1, j=1,2, \ldots, m_{i}, i=1,2,3\right\} \cup\left\{u_{x_{i 1}}: i=1,2,3\right\}$ is a complete set of extreme functions for $C\left(m_{1}, m_{2}, m_{3}\right)$.

(c) Suppose $k=2$. If $m_{1}=m_{2}=1$, then $s_{11} \sim s_{21} \sim u_{1}$ is the unique extreme function. Otherwise, $\left\{s_{i j}: j=1,2, \ldots, m_{i}>1, i=1,2\right\}$ is a complete set of extreme functions for $C\left(m_{1}, m_{2}\right)$.

Proof. Take $f \in \mathcal{S}$ in normal form. If all values of $f$ are nonpositive, then we can directly write $f$ as a linear combination of the functions $s_{i j}$ with nonnegative coefficients. If not all values of $f$ are nonpositive, suppose that $\alpha$, the maximum value of $f$, occurs at an element in $C_{i}$. Then, since $f$ is supermodular, its value on all other chains must be less than or equal to $-\alpha$. By subtracting $\alpha$ times the normal form of $u_{x_{i 1}}$, as given in the proof of Lemma 3.1, we obtain a function with all values nonpositive. This proves (a), and for (b) and (c) we use the results from the corresponding statements in Theorem 3.2.

\section{The Lattice $Z_{n}^{k}$}

Generally, one considers the supermodular ordering between random variables defined on $\mathcal{R}^{k}$, that is, on $k$-variate random variables. Therefore, in our context of finite lattices, we are interested in supermodular functions on the lattice $Z_{n}^{k}$ that consists of all $k$-tuples $x=\left(x_{1}, x_{2}, \ldots, x_{k}\right)$, in which each $x_{i}$ takes a value of 0 to $n-1$ and $x \leq y$ if $x_{i} \leq y_{i}$ for all $i=1,2, \ldots, k$.

In this section, we first give a basis for the modular functions on $Z_{n}^{k}$. We then determine when the supermodular functions $u_{F}$ and $\ell_{G}$ are extreme. After that, we provide a useful representation of functions in $\mathcal{F}$ and use that representation to find complete sets of extreme functions for the lattices $Z_{n}^{2}$ and $Z_{2}^{3}$.

\subsection{MODULAR FUNCTIONS ON $Z_{n}^{k}$}

For the purpose of finding a basis for the modular functions, define $\ell$ and $n$, respectively, on $Z_{n}^{k}$ by $\ell(x)=\sum_{i=1}^{k} x_{i}$ and by setting $n(x)$ equal to the number of nonzero entries in $x$. Let $\delta(i, m)$ denote the element of $Z_{n}^{k}$ that consists of all 
zeros except for an entry of $m$ in position $i$. Note that $\delta(i, 0)=0$ for all $i$. For $i=1,2, \ldots, k$ and $m=1,2, \ldots, n-1$, let $M(i, m)$ denote the function that takes the value 1 on all $x$ for which $x_{i}=m$ and takes the value 0 elsewhere. It is clear that each $M(i, m)$ is modular and that these functions are linearly independent.

THEOREM 4.1. For the lattice $Z_{n}^{k}$, the space $\mathcal{M}$ of modular functions is generated by $M(i, m)$ and the constant functions and is, therefore, of dimension $k(n-1)+1$.

Proof. Let $f$ be a modular function that is 0 at 0 . We will prove the theorem by showing that $f$ is determined by its values on $\delta(i, m)$ for $i=1,2, \ldots, k$ and $m=1,2, \ldots, n-1$. Specifically, we will show that if $x=\left(x_{1}, x_{2}, \ldots, x_{k}\right) \neq 0$, then

$$
f(x)=\sum_{i=1}^{k} f\left[\delta\left(i, x_{i}\right)\right] .
$$

We verify (4.1) by induction on $\ell(x)$. Expression (4.1) is clearly true for elements $y$ such that $\ell(y)=1$. Suppose it true for all $y$ with $\ell(y)<r$, and choose $x$ with $\ell(x)=r$. If $n(x)=1$, then (4.1) is trivially true. If not, let $x^{\prime}$ be obtained from $x$ by replacing a nonzero entry with 0 , and let $x^{\prime \prime}$ be obtained by replacing all the other entries with 0 . Then, $x^{\prime} \vee x^{\prime \prime}=x, x^{\prime} \wedge x^{\prime \prime}=0$, and

$$
f(x)=f\left(x^{\prime}\right)+f\left(x^{\prime \prime}\right)
$$

because $f$ is modular. Because both $x^{\prime}$ and $x^{\prime \prime}$ satisfy (4.1) by the induction hypothesis, it is clear that $x$ does as well.

One can also derive Theorem 4.1 from Theorem 3.3 of Topkis [12], which characterizes the modular functions for any lattice that is a product of chains. Note that Theorem 4.1 implies the well known fact that if $X$ and $Y$ are random variables on $Z_{n}^{k}$ ordered according to the supermodular order, then they have equal marginal distributions. In the particular case of $Z_{2}^{k}$ viewed as the lattice of subsets of a $k$-element set, the modular functions are sums of a signed measure on the set plus a constant.

\subsection{WHEN ARE $u_{F}$ AND $\ell_{G}$ EXTREME SUPERMODULAR FUNCTIONS ON $Z_{n}^{k}$ ?}

From Theorem 2.9, we know that $u_{F}$ and $\ell_{G}$ (see Definitions 2.6 and 2.8) are supermodular for fans $F$ and $G$ on any finite lattice. In Lemma 3.1 and Theorem 3.2, we determined when $u_{x}$ and ind $d_{x}$ on chain lattices are extreme. In 
this subsection, as a parallel to that, we determine when $u_{F}$ and $\ell_{G}$ are extreme on $Z_{n}^{k}$.

Define $\left(Z_{n}^{k}\right)^{*}=\left\{x \in Z_{n}^{k}: x_{i} \neq 0\right.$ for at least 2 values of the index $i=1$, $2, \ldots, k\}$. In other words, $\left(Z_{n}^{k}\right)^{*}$ consists of elements other than $\delta(i, m)$ and 0 . We say that a function $f$ on $Z_{n}^{k}$ is in normal form if $f(x)=0$ for all $x \notin\left(Z_{n}^{k}\right)^{*}$. Note that we do not have a general definition of normal form; it depends on the particular lattice. The definition we give for normal form for functions on $Z_{n}^{k}$ is different from the one we gave for chain lattices in Section 3.

LEMMA 4.2. For all $f \in \mathcal{F}$, there is an $f^{o}$ in normal form such that $f \sim f^{o}$, in which $f^{o}$ is unique up to positive scalar multiple.

Proof. Let

$$
f^{o}=f-\sum_{i, m \neq 0} f(\delta(i, m)) M(i, m)-f(0) I,
$$

in which $I$ is the function that is identically 1 . It is clear that $f^{o}$ is in normal form and is equivalent to $f$. If $g$ is any other such function, then $f^{o}-\alpha g$ is modular for some $\alpha>0$ and takes the value 0 on all $\delta(i, m)$ and on 0 . By Theorem 4.1, $f^{o}=\alpha g$.

LEMMA 4.3. If $f \in \mathcal{S}$ is in normal form, then $f$ is order-preserving. That is, $x \leq y$ implies that $f(x) \leq f(y)$.

Proof. Suppose that $x$ and $y$ are elements of $Z_{n}^{k}$ that agree in all positions, except that $x_{i}=m$ and $y_{i}=m+1$ for some $m=0,1, \ldots, n-1$. Then, by supermodularity of $f$,

$$
f(y) \geq f(x)+f[\delta(i, m+1)]-f[\delta(i, m)]=f(x) .
$$

An obvious induction completes the proof.

From Lemmas 4.2 and 4.3, we can deduce the well known result that in the definition of supermodular order (1.2), we can restrict $f$ to be an increasing supermodular function in our finite setting. See Müller and Stoyan (9; Theorem 3.9.11) for the corresponding result in a more general setting.

We say $x \in Z_{n}^{k}$ is $f$-zero if $f^{o}(x)=0$, in which $f^{o} \sim f$ is in normal form. It follows from Lemma 4.3 that if $x$ is $f$-zero, then $y$ is $f$-zero for all $y \leq x$.

LEMMA 4.4. Suppose $f \preceq g$, where $f$ and $g \in \mathcal{S}$. If $x$ is $g$-zero, then it is $f$-zero.

Proof. Suppose $f$ and $g$ are in normal form. If $x$ is $g$-zero but not $f$-zero, let $z$ be a minimal point from those elements dominated by $x$ on which $f$ takes a nonzero value. Then, $z$ has at least two nonzero entries, so we can certainly find nonzero $a, b$ such that $z=a \vee b$. But, then, $\delta_{f}(a, b)=f(z) \neq 0$, a contradiction since $(a, b)$ is $g$-modular by Lemma 4.3. Recall that $\delta_{f}$ is defined in (2.1). 
THEOREM 4.5. Let $F$ be an upper fan in $Z_{n}^{k}$; see Definition 2.6.

(a) If $F=\left\{a_{1}\right\}$, then $u_{F}$ is extreme if and only if $a_{1}$ has more than one nonzero coordinate, and $\ell_{F}$ is extreme if and only if $a_{1}$ has fewer than $(k-1)$ nonzero coordinates. Otherwise, $u_{F}$ and $\ell_{F}$ are modular.

(b) If $F=\left\{a_{1}, a_{2}\right\}$ is an upper fan of length 2 , then $u_{F}=u_{a_{1}}+u_{a_{2}}$; thus, $u_{F}$ is not extreme. Similarly, if $G=\left\{a_{1}, a_{2}\right\}$ is a lower fan of length 2, then $\ell_{G}=\ell_{a_{1}}+\ell_{a_{2}}$; thus, $\ell_{G}$ is not extreme.

(c) For any upper fan $F$ or lower fan $G$ of length greater than $2, u_{F}$ and $\ell_{G}$ are extreme.

Proof. We prove this theorem for $u_{F}$. Parts (a) and (b) are straightforward, so we focus on part (c). Suppose $f \in \mathcal{S}$ is in normal form and $f \preceq u_{F}$. We will show that $f$ is a nonnegative multiple of $u_{F}$. The multiple, of course, could be 0 , which arises when $f$ is modular.

Let $F=\left\{a_{1}, a_{2}, \ldots, a_{m}\right\}$ be an upper fan with $m \geq 3$, and let $b$ be the common least upper bound of any pair. Let $f\left(a_{1}\right)=\alpha$. For each $i$, we know that $\left(a_{1}, a_{i}\right)$ is $u_{F}$-modular and, thereby, $f$-modular. Moreover $a_{1} \wedge a_{i}$ is $u_{F}$-zero, so $f\left(a_{1} \wedge a_{i}\right)=0$ by Lemma 4.4. Therefore, $f(b)=\alpha+f\left(a_{i}\right)$ for all $i$. Similarly, $f(b)=f\left(a_{2}\right)+f\left(a_{3}\right)$, showing that for all $i, f\left(a_{i}\right)=\alpha$ and $f(b)=2 \alpha$.

Suppose that $z$ is greater than or equal to some $a_{i}$ but not $b$. Let $w$ be such that $a_{i} \vee w=z$ and $a_{i} \wedge w=0$; the existence of such an element $w$ is easily verified. Our hypothesis on $z$ shows that $w$ cannot dominate any of the elements in $F$; therefore, $w$ is $u_{F}$-zero, from which it follows that $f(w)=0$. Thus, $\left(a_{i}, w\right)$ is $u_{F}$ modular and, thereby, $f$-modular, showing that $f(z)=\alpha$. A similar argument shows that for all $z$ greater than or equal to $b, f(z)=2 \alpha$. Thus, we have shown that $f=\alpha u_{F}$.

\subsection{A REPRESENTATION OF FUNCTIONS IN $\mathcal{F}$}

We now introduce a representation of functions that will be useful in determining extreme supermodular functions. At the end of this subsection, we describe how to thus use this representation. In the next subsection, we apply our technique and find complete sets of extreme supermodular functions for $Z_{n}^{2}$ and $Z_{2}^{3}$.

For any $x \in\left(Z_{n}^{k}\right)^{*}$, let $\phi_{x}$ be the function defined by

$$
\phi_{x}(y)=\left\{\begin{array}{rr}
1, & \begin{array}{rl}
\text { if } y_{i}=x_{i} \text { except for an even number } \\
& \text { of indices } j \text { on which } y_{j}=x_{j}-1 ; \\
-1, & \text { if } y_{i}=x_{i} \text { except for an odd number } \\
& \text { of indices } j \text { on which } y_{j}=x_{j}-1 ;
\end{array} \\
0, \quad \text { for all other } y .
\end{array}\right.
$$

For example, take $k=3$ and $n \geq 5$. Then, $\phi_{234}$ takes the value 1 on 234, 124, 133, and 223; the value -1 on $134,224,233$, and 123 ; and the value 0 elsewhere. 
THEOREM 4.6.

$$
<u_{y}, \phi_{x}>= \begin{cases}1, & \text { if } y=x \\ 0, & \text { if } y \neq x\end{cases}
$$

Here $<\cdot, \cdot>$ denotes the inner product on $\mathcal{F}$ defined by $\langle f, g\rangle=\sum_{x \in Z_{n}^{k}}$ $f(x) g(x)$.

Proof. When $y=x$, this is clear because there is no other element in the support of $\phi_{x}$ that is greater than or equal to $x$, other than $x$ itself. In all other cases, the set of elements in the support of $\phi_{x}$ that are greater than $y$ either is empty or contains an even number of elements, with half of them assigned the value of 1 and the other half -1 .

Given $f \in \mathcal{F}$, define $\hat{f}$ on $\left(Z_{n}^{k}\right)^{*}$ by $\hat{f}(x)=<f, \phi_{x}>$. We refer to $\hat{f}$ as the upper transform of $f$. From Theorem 4.6, if $f$ is in normal form, then we can write

$$
f=\sum_{x \in\left(Z_{n}^{k}\right)^{*}} \hat{f}(x) u_{x}
$$

If $f \sim g$, then $\hat{f}=\alpha \hat{g}$ for some $\alpha>0$; therefore, the upper transform (up to positive scalar multiple) is independent of the equivalence class.

Moreover, the following is clear from the definitions of $\hat{f}$ and $\phi_{x}$ :

$$
\text { If } f \in \mathcal{S} \text { and if } x \in Z_{n}^{k} \text { has exactly two nonzero entries, then } \hat{f}(x) \geq 0 \text {. }
$$

Indeed, in that case, $\hat{f}(x)=\delta_{f}(y, z)$ for some $y, z$. For example, if $x=(a, b$, $0, \ldots, 0)$, we can take $y=(a-1, b, 0, \ldots, 0)$ and $z=(a, b-1,0, \ldots, 0)$.

The inverse formula for $f$ in normal form is given simply as

$$
f(x)=\sum_{y \leq x, y \in\left(Z_{n}^{k}\right)^{*}} \hat{f}(y),
$$

for $x \in\left(Z_{n}^{k}\right)^{*}$. Also, from Theorem 4.6, we have that

$$
\hat{u}_{y}(x)= \begin{cases}1, & \text { if } y=x \\ 0, & \text { otherwise }\end{cases}
$$

The following lemma extends this result to $\hat{u}_{F}$, for $F$ an upper fan of length at least two. We omit the proof because the result follows easily from the inverse formula in (4.4).

LEMMA 4.7. Suppose $F=\left\{a_{1}, a_{2}, \ldots, a_{m}\right\}$ is an upper fan of length $m \geq 2$ with $a_{i} \in\left(Z_{n}^{k}\right)^{*}$ for $i=1,2, \ldots, m$ and with common pairwise least upper bound 
$b$, then $\hat{u}_{F}$ is given by

$$
\hat{u}_{F}(y)= \begin{cases}2-m, & \text { if } y=b \\ 1, & \text { if } y=a_{i} \text { for some } i=1,2, \ldots, m \\ 0, & \text { otherwise }\end{cases}
$$

To help deduce supermodularity of $f \in \mathcal{F}$ from $\hat{f}$, we have the following theorem.

THEOREM 4.8. For any incomparable $x, y \in Z_{n}^{k}$ and for $f \in \mathcal{F}$ in normal form,

$$
\delta_{f}(x, y)=\sum_{z \in K_{x, y}} \hat{f}(z)
$$

in which

$$
K_{x, y}=\left\{z \in\left(Z_{n}^{k}\right)^{*}: z \leq x \vee y, z \not \leq x, z \not \leq y\right\} .
$$

Proof. The set $\{z \leq x \vee y\}$ is a union of four disjoint sets, namely (1) $K_{x, y}$, (2) those elements that are $\leq x$ but $\not \leq y$, (3) those elements that are $\leq y$ but $\not \leq x$, and (4) those elements that are $\leq x \wedge y$. Since $f(x)$ is the sum of the values of $\hat{f}$ over the second and fourth sets, $f(y)$ is the sum of these values over the third and fourth, and $f(x \wedge y)$ is the sum of these values over the fourth, the result follows.

Similarly to Lemma 4.4 , we have

LEMMA 4.9. If $f \preceq g$ and $\hat{g}(x)=0$, then $\hat{f}(x)=0$.

Proof. Suppose that there is some point $z$ with $\hat{g}(z)=0$ but $\hat{f}(z) \neq 0$. Choose such a $z$ that is minimal. Let $A=\left\{x \in\left(Z_{n}^{k}\right)^{*}: x \leq z\right.$, and $\left.\hat{g}(x) \neq 0\right\}$. If $A$ is not empty, let $a=\max (A)$. Choose incomparable points $v, w$ such that $z \geq$ $w \geq a$ and $v \vee w=z$. It is straightforward to check that such points exists. If $b \in K_{v, w}$, then $b \leq z$ and $b \not \leq a$ imply that $b \notin A$ and so $\hat{g}(b)=0$. By Theorem $4.8,(v, w)$ is $g$-modular. Therefore, $(v, w)$ is $f$-modular, and the sum of the values of $\hat{f}$ on $K_{v, w}$ is zero. But this is a contradiction, since $f(z) \neq 0$ and for all other points $b$ in $K_{v, w}, \hat{f}(b)=0$ by minimality. If $A$ is empty, we can choose any incomparable $(v, w)$ such that $v \vee w=z$ and argue as above.

The use of the upper transform facilitates the search for extreme (supermodular) functions and gives us immediate answers in a couple of simple cases. We first describe a general strategy that will not only prove that a certain set is extreme but can provide an algorithm for writing a function as a positive linear combination of proposed extreme functions. 
Let $\mathcal{E}$ be a potential set of extreme functions. Let $\mathcal{G}$ be the set of all $f$ with $\hat{f} \geq 0$, so that any function in $\mathcal{G}$ is equivalent to a positive linear combination of upper functions by (4.2) and Lemma 4.2. Suppose we can find a closed cone (in the usual topology on $\left.\mathcal{R}^{d}\right) \mathcal{K} \supseteq \mathcal{S}$ such that for all $f$ in $\mathcal{K}$ but not in $\mathcal{G}$, there is an $e \in \mathcal{E}$ and $\alpha>0$ with $f-\alpha e \in \mathcal{K}$. By taking the maximum value of $\alpha$ such that $f-\alpha e \in \mathcal{K}$, which exists because $\mathcal{K}$ is closed, and repeating, we will eventually write $f$ as a sum of an element in $\mathcal{G}$ and a positive linear combination of elements in $\mathcal{E}$. This follows from the cone structure of $\mathcal{K}$. For example, if $\alpha_{1}$ is the maximum value such that $f-\alpha e_{1} \in \mathcal{K}$ and $\alpha_{2}$ is the maximum value such that $f-\alpha_{1} e_{1}-\alpha_{2} e_{2} \in \mathcal{K}$, then we cannot choose $e_{1}$ again as the next function to subtract a multiple of, for if $f-\left(\alpha_{1}+\epsilon\right) e_{1}-\alpha_{2} e_{2} \in \mathcal{K}$ for some $\epsilon>0$, we could add $\alpha_{2} e_{2}$ to deduce that $f-\left(\alpha_{1}+\epsilon\right) e_{1} \in \mathcal{K}$, thereby contracting the maximality of $\alpha_{1}$. The trick is to come up with a suitable $\mathcal{K}-$ or we should say, with a suitable description of $\mathcal{K}$ - to fit the situation, since a corollary of the above is that in fact $\mathcal{K}=\mathcal{S}$.

\subsection{APPLYING THE REPRESENTATION TO $Z_{n}^{2}$ AND $Z_{2}^{3}$}

In this subsection, we apply the method described at the end of the previous subsection to find complete sets of extreme supermodular functions on $Z_{n}^{2}$ and $Z_{2}^{3}$.

THEOREM 4.10. A complete set of extreme functions for the lattice $Z_{n}^{2}$ is given by $\left\{u_{x}: x \in\left(Z_{n}^{2}\right)^{*}\right\}$.

Proof. By (4.3), $\mathcal{S} \subseteq \mathcal{G}$, and we are done.

From Theorem 4.10, we can deduce the well known result that for bivariate distributions, the supermodular order is equivalent to the upper orthant order in our finite setting. See Müller and Stoyan (9; Theorem 3.8.2) for this result in a more general setting.

We next consider the lattice $Z_{2}^{3}$, which we view as the lattice of all subsets of the set $\{a, b, c\}$.

THEOREM 4.11. For the lattice $Z_{2}^{3}$, the extreme functions are all of the form $u_{F}$, in which $F$ is an upper fan from the list $\{a b, a c, b c\},\{a b\},\{a c\},\{b c\}$, and $\{a b c\}$.

Proof. Here we can directly take $\mathcal{K}=\mathcal{S}$. Let $f \in \mathcal{S}$. If $f \notin \mathcal{G}$, then $\hat{f}(a b c)<$ 0 because $\hat{f} \geq 0$ on any two-element subset of $\{a, b, c\}$ by supermodularity of $f$, or equivalently, by (4.3). Since $K_{b c, a c}=\{a b c, a b\}$, we must have $\hat{f}(a b)+$ $\hat{f}(a b c) \geq 0$ by Theorem 4.8, and similarly both $\hat{f}(a c)+\hat{f}(a b c) \geq 0$ and $\hat{f}(b c)+\hat{f}(a b c) \geq 0$.

It is easily seen that

$$
u_{a b, a c, b c}=u_{a b}+u_{a c}+u_{b c}-u_{a b c} .
$$


From (4.2) and (4.6), we deduce that $\hat{u}_{a b, a c, b c}$ takes the value of 1 on each twoelement set and the value -1 on $a b c$. Let $h=f+\hat{f}(a b c) u_{a b, a c, b c}$; then, $\hat{h}=\hat{f}+\hat{f}(a b c) \hat{u}_{a b, a c, b c}$ takes the value of 0 on $a b c$. Moreover, on each twoelement set $A, \hat{h}$ takes the value $\hat{f}(A)+\hat{f}(a b c)$, which we have shown is nonnegative. It follows that $h \in \mathcal{G}$ as required.

Remark. For the extreme functions on $Z_{2}^{3}$, we do not need functions of the form $\ell_{G}$, in which $G$ is a lower fan, because each $\ell_{G}$ is equivalent to some $u_{F}$. Indeed, it is easy to see that $\ell_{a} \sim u_{b c}, \ell_{a, b, c} \sim u_{1}$, and $\ell_{0} \sim u_{a b, a c, b c}$. This correspondence does not occur, however, for $Z_{2}^{k}$ when $k>3$.

An alternative proof which does not use the upper transform is to simply note that for any $f \in \mathcal{S}$ in normal form, satisfying $f(a b) \geq f(a c) \geq f(b c)$,

$$
\begin{aligned}
f= & f(b c) u_{a b, a c, b c}+[f(a b)-f(b c)] u_{a b}+[f(a c)-f(b c)] u_{a c}+[f(a b c) \\
& -f(a b)-f(a c)] u_{a b c} .
\end{aligned}
$$

Note that the supermodularity and normalcy of $f$ imply that $f(b c) \geq 0$.

Conjecture. We conjecture that for $Z_{2}^{k}, k \neq 4$, any extreme function is of the form $u_{F}$ or $\ell_{G}$. The case $k=4$ is different because any two-element set is quasiindecomposable. We will consider this lattice in detail in the next section.

\section{The Lattice $Z_{2}^{4}$}

In this section, we determine a complete set of extreme supermodular functions for the lattice $Z_{2}^{4}$. Similarly as we did for $Z_{2}^{3}$, we view $Z_{2}^{4}$ as the lattice of subsets of the set $\{a, b, c, d\}$.

LEMMA 5.1. $q$ qin $_{a b}$ is extreme in $Z_{2}^{4}$.

Proof. Suppose that $f \preceq q i n_{a b}$ is in normal form. From the $q i n_{a b}$-modularity of $(a, b)$, it follows that $f(a b)=0$. Let $f(a c)=\alpha$. Then, by the $q i_{a b}$-modularity of any non-disjoint pair of two-element sets (not including $a b$ ), we see that the value of $f$ on all two-element sets is $\alpha$, and the value of $f$ on all three-element sets is $2 \alpha$. Finally, the $q_{i n}$-modularity of $(a b c, a b d)$ shows that $f(a b c d)=4 \alpha$. Since the normal form of $q_{i n}$ takes the value of 4 at 1,2 on the three-element sets, 1 on the two-element sets other than $a b$, and zero elsewhere, we are done.

Our main result in this section is the following theorem.

THEOREM 5.2. A complete set of extreme functions for the lattice $Z_{2}^{4}$, viewed as the lattice of subsets of a four-element set, consists of the following: $\ell_{0} ; \ell_{B}$, in which $B$ is a lower fan consisting of three one-element sets; qin $_{A}$, in which $A$ is a 
two-element set; $u_{F}$ for the upper fans $F$ in Example 2.7 (iii) - (vi); and $u_{x}$, in which $x$ is a subset of $\{a, b, c, d\}$ with more than one element.

Remark. We do not need to include the other functions of the form $\ell_{G}$ since they are all equivalent to listed ones. For example, $\ell_{a b} \sim u_{c d}, \ell_{\{a b, a c, a d\}} \sim u_{b c d}$, $\ell_{\{a b, c, d\}} \sim u_{\{a b, a c d, b c d\}}$, and $\ell_{\{a, b, c, d\}} \sim u_{\{a b c, a b d, a c d, b c d\}}$.

Before giving the proof, we need some preliminary comments. We will need the values of $\hat{f}$ for the given functions. For $u_{F}$, where $F$ is an upper fan, we have given $\hat{u}_{F}$ in Lemma 4.7. For the other three functions in the statement of Theorem 5.2, we represent $\hat{f}$ as eleven-dimensional vectors, where the first value is $\hat{f}(a b c d)$, the next four are the values of $\hat{f}$ on the three-element sets, in the order $a b c, a b d, a c d, b c d$, and the final six are the values of $\hat{f}$ on the two-element sets, in the order $a b, a c, b c, a d, b d, c d$.

$$
\begin{aligned}
\operatorname{qin}_{c d} & =(1,-1,-1,0,0,1,1,1,1,1,0), \\
\hat{\ell}_{0} & =(1,-1,-1,-1,-1,1,1,1,1,1,1), \\
\hat{\ell}_{b, c, d} & =(-1,0,0,0,1,1,1,0,1,0,0) .
\end{aligned}
$$

We follow the strategy given in the preceding section. We let $\mathcal{K}$ consist of all $f$ such that $\delta_{f}(A, B) \geq 0$ if $A$ and $B$ are both three-element sets, both twoelement sets that are not disjoint, or both one-element sets. Clearly $\mathcal{K} \supseteq \mathcal{S}$. By using Theorem 4.8, we can translate this into conditions on $\hat{f}$. Consider any "diamond" consisting of $a b c d$, two three-element sets $B$ and $C$, and $B \cap C$. Then, $f$ is in $\mathcal{K}$ if and only if $\hat{f}$ is nonnegative on $B \cap C$ (the base of the diamond), the sum of the two values of $\hat{f}$ on any "lower edge" is nonnegative, and the sum of the values of $\hat{f}$ on all four points is nonnegative. To see this, suppose, for example that $B=a b c$ and $C=a b d$. Then, $\{a b\}=K_{a, b}$, the two points on a lower edge $\{a b, a b c\}=K_{a c, b c}$, and the set of all four points $\{a b c d, a b c, a b d$, $a b\}=K_{a c d, b c d}$.

We will often want to show that such a sum of values is strictly positive. Note that to show that the sum of all four values is positive for $f \in \mathcal{K}$, we need only show the sum of the two values on any "upper edge" is positive.

For any function $f$ and any two-element set $A$, let $\gamma_{f}(A)=-(\hat{f}(a b c d)+$ $\hat{f}(B)+\hat{f}(C)$, in which where $B$ and $C$ are the two three-element sets containing $A$. Then, the fact that the sum of the four values of $\hat{f} \geq 0$ means that

$$
\gamma_{f}(A) \leq \hat{f}(A) .
$$

Proof of Theorem 5.2. Given any $f$ in $\mathcal{K}$ but not in $\mathcal{G}$, we seek $e$ from the list of extreme functions given in the statement of Theorem 5.2 such that $f-\alpha e \in \mathcal{K}$ for some $\alpha>0$. This will occur if and only if we can find $e$ such that whenever the sum of $\hat{e}$ over one of the subsets $\mathbf{S}$ (equal to a diamond, lower edge, or base) 
is positive, then the sum of the values of $\hat{f}$ over $\mathbf{S}$ is positive. We can, then, choose

$$
\alpha=\min _{\mathbf{S}} \frac{\sum_{y \in \mathbf{S}} \hat{f}(y)}{\sum_{y \in \mathbf{S}} \hat{e}(y)} .
$$

We will assume throughout, relabeling if necessary, that

$$
\hat{f}(a b c) \leq \hat{f}(a b d) \leq \hat{f}(a c d) \leq \hat{f}(b c d) .
$$

Note that for $e=u_{a b, a c, b d}, u_{a b c, a b d, a c d, b c d}, \ell_{0}$, or $\ell_{b, c, d}$, the sum of values of $\hat{e}$ over any diamond equals 0 , and in order to verify that one of these will work, we need only check the condition for $\hat{f}$ on the base and lower edges.

Let $\iota(f)$ be the number of three-element sets with $\hat{f}(B)<0$.

CASE 1: $\iota(f) \geq 3$. Choose $e=\ell_{0}$. The sum for $\hat{\ell}_{0}$ is positive only on the diamond bases, that is, the two-element sets. By virtue of the fact that any twoelement set is contained in one of $a b c, a b d$, or $a c d$, on which $\hat{f}$ is negative, the edge condition implies that $\hat{f}(A)>0$ on all two-element sets. By "edge condition," we mean that the sum of the values of $\hat{f}$ is nonnegative on the lower edges of any diamond.

CASE 2: $\iota(f)=2$. As in Case 1, we must have $\hat{f}(A)>0$ for all two-element sets, except possibly $c d$. Therefore, the sum of $\hat{f}$ is positive on any lower edge for which the two-element set is other than $c d$ and the three-element set is either $a c d$ or $b c d$. If also $\hat{f}(a b)+\hat{f}(a b d)>0$, we can choose $e=u_{a b, a c, b c}$.

If $\hat{f}(a b)+\hat{f}(a b d)=0$, we must have $\hat{f}(a b c d)=-\hat{f}(a b c)=-\hat{f}(a b d)>0$ in order that the sum over the diamond with base $a b$ is nonnegative. Then, the sum of $\hat{f}$ over all diamonds is positive, as seen from looking at the "right" upper edge. In this case, we can take $e=q i n_{c d}$.

CASE 3: $\iota(f)=1$. Because $\hat{f}(a b c)+\hat{f}(a b) \geq 0$ and $\hat{f}(a b c)<0$, we have $\hat{f}(a b)>0$. Similarly, $\hat{f}(a c)>0$ and $\hat{f}(b c)>0$. It follows that we can take $e=u_{a b, a c, b c}$.

CASE 4: $\iota(f)=0$. We divide this case into four subcases, A $-\mathrm{D}$. Note that since $f \notin \mathcal{G}$, we must have $\hat{f}(a b c d)<0$.

SUBCASE A: Suppose $\gamma_{f}(b c)>0$. This implies by (5.2) that $\gamma_{f}(a b)$ and $\gamma_{f}(a c)$ are positive because $\gamma_{f}(a b) \geq \gamma_{f}(a c) \geq \gamma_{f}(b c)>0$. It follows from (5.1) that $\hat{f}(a b), \hat{f}(a c)$, and $\hat{f}(b c)>0$. We can, therefore, take $e=u_{a b, a c, b c}$ since the 
lower edges on which $\hat{e}$ has a positive sum are only those from $a b, a c$, or $b c$ to a point other than $a b c$.

SUBCASE B: Suppose $\gamma_{f}(b c) \leq 0$ and $\gamma_{f}(a d)>0$. Then, by (5.2), $\gamma_{f}(a b) \geq \gamma_{f}(a c) \geq \gamma_{f}(a d)>0$, and it follows from (5.1) that $\hat{f}(a b), \hat{f}(a c)$, and $\hat{f}(a d)$ are positive. Moreover, $\hat{f}(b c d)>0$ since if not, then $\hat{f}(B)=0$ for all three-element sets $B$ by (5.2), and we would have $\gamma_{f}(b c)=-\hat{f}(a b c d)>0$, a contradiction. We can take $e=\ell_{b, c, d}$ because the sums of $\hat{e}$ are positive over all lower edges starting from $a b, a c, a d$ or ending with $b c d$.

SUBCASE C: Suppose $\gamma_{f}(b c) \leq 0, \gamma_{f}(a d) \leq 0$, and $\gamma_{f}(a b)>0$. Then, $\hat{f}(a c d), \hat{f}(b c d)$, and $\hat{f}(a b)$ are all positive. Indeed, if $\hat{f}(a c d)=0$, then $\gamma_{f}(a d)$ $=-\hat{f}(a b c d)>0$, a contradiction. Also, the sum of $\hat{f}$ over the four elements of the diamond $\{a b c d, a c d, b c d, a d\}$ is positive. Indeed, $\hat{f}(a b c)+\hat{f}(a b d)<$ $-\hat{f}(a b c d)$ because $\gamma_{f}(a b)>0$, while $\hat{f}(a b c)+\hat{f}(b c d) \geq-\hat{f}(a b c d)$ because $\gamma_{f}(b c) \leq 0$. It follows that $\hat{f}(b c d)>\hat{f}(a b d)$, so $\hat{f}(b c d)+\hat{f}(a c d)>\hat{f}(a b d)+$ $\hat{f}(a c d) \geq-\hat{f}(a b c d)>0$, where the next-to-the-last inequality arises from $\gamma_{f}(a d) \leq 0$. In this case, we can take $e=u_{a b, a c d, b c d}$.

SUBCASE D: Suppose $\gamma_{f}(a b) \leq 0$. If $\hat{f}(a b c)>0$, then the sum of values of $\hat{f}$ over any lower edge is positive, and we can choose $e=u_{a b c, a b d, a c d, b c d}$.

If $\hat{f}(a b c)=0$, then $\hat{f}(a b d)>0$. Indeed, if $\hat{f}(a b d)=0$, then $\gamma_{f}(a b)=$ $-\hat{f}(a b c d)>0$, a contradiction. The sum of values of $\hat{f}$ is positive over any diamond with base equal to $a d, b d$, or $c d$. Also, the sum of the values of $\hat{f}$ is positive on any lower edge ending in $a b d, a c d$, or $b c d$. Thus, we can choose $e=u_{a b d, a c d, b c d}$.

\section{The Lattice $Z_{n}^{3}$}

In this section, we examine extreme supermodular functions on the lattice $Z_{n}^{3}$. We begin by introducing a new collection of supermodular functions, and we determine when those functions are extreme. We then describe a complete set of extreme supermodular functions on $Z_{3}^{3}$.

\subsection{MULTIFANS IN $Z_{n}^{3}$}

In this subsection, we define a new collection of functions. In the next subsection, we show that members of that collection are supermodular, and we determine when they are extreme.

For each $x \in Z_{n}^{3}$ and $i=1,2,3$, let $C(i, x)$ denote the set of all points $y$ whose $i$-th coordinate is less than or equal to $x_{i}$ and whose other two coordinates agree 
with $x$. For example, if $x=(3,2,4)$, then $C(1, x)=\{(3,2,4),(2,2,4),(1,2,4)$, $(0,2,4)\}$. We will refer to any such set $C(i, x)$ as a line segment.

A multifan consists of a pair of nonempty subsets of $\left(Z_{n}^{3}\right)^{*},(A, B)$, such that

(i) Each of $A$ and $B$ intersects any line segment in at most one element.

(ii) For each $a \in A$ and $i=1,2,3$, the set $C(i, a)$ intersects $B$ in a point $p_{i}(a)$, which is necessarily unique from (i).

(iii) For each $b \in B$, there exists some $a \in A$ and index $i=1,2,3$ such that $b=p_{i}(a)$.

Note that the $i$ and $a$ in (iii) need not be unique.

If $(A, B)$ is a multifan with $A \cap B$ non-empty, then $A=B=\{x\}$, and we define $u_{A, B}$ to be the upper function $u_{x}$. If $(A, B)$ is a multifan with $A \cap B=\phi$, then we define the function $u_{A, B}$ (modulo the modular functions) to be the one with

$$
\hat{u}_{A, B}(x)= \begin{cases}1, & x \in B \\ -1, & x \in A \\ 0, & \text { elsewhere }\end{cases}
$$

Recall that we can recover $u_{A, B}$ from $\hat{u}_{A, B}$ by the inverse formula in (4.4). When $A$ has one point, it is easy to verify that either $B=A$ or $B$ is an upper fan of length three, with $u_{A, B}=u_{B}$.

A multifan will be called irreducible if it is not possible to find a partition of $A$ into two sets $A_{1}$ and $A_{2}$ and a partition of $B$ into two sets $B_{1}$ and $B_{2}$ such that $\left(A_{1}, B_{1}\right)$ and $\left(A_{2}, B_{2}\right)$ are multifans. Note that if $(A, B)$ is a multifan with $A=\{x\}$, then it is automatically irreducible.

EXAMPLE 6.1. We list the irreducible multifans in the lattice $Z_{3}^{3}$.

1. $|A|=1$ : For each element $A=\{(a, b, c)\}$, for which no entry is equal to zero, we have a collection of $2^{k}$ fans, in which $k$ is the number of coordinates of $(a, b, c)$ equal to 2. Each $B$ is of the form $B=\{(x, b, c),(a, y, c),(a, b, z)\}$, in which each $x, y$, and $z$ is either 0 or 1 if the corresponding entry above it in $A$ is 2 , or 0 if the corresponding entry above it in $A$ is 1 . We also have all the multifans for which $A=B=\{x\}$, with $x \in\left(Z_{3}^{3}\right)^{*}$.

2. $|A|=2$ :

a. $A=\{(1,2,2),(2,1,2)\}$, and $B=\{(0,2,2),(2,0,2),(1,1,2),(2,1, x),(1$, $2, y)\}$, in which each of $x$ and $y$ is either 0 or 1 , so there are four possibilities for $B$ corresponding to this particular $A$. Then, there are another two possibilities for $A$ obtained by permutation, with four $B s$ each. b. $A=\{(1,1,2),(1,2,1)\}$, and $B=\{(0,1,2),(1,0,2),(0,2,1),(1,2,0),(1$, $1,1)\}$, with two other possibilities obtained by permutation. 
3. $|A|=3$ :

a. $A=\{(1,2,2),(2,1,2),(2,2,1)\}$. In all cases, $B$ will contain $(0,2,2),(2$, $0,2),(2,2,0)$, and either (i) $(1,1,2),(1,2,1)$, and $(2,1,1)$, (ii) $(1,1,2)$, $(2,1,1),(0,2,1)$, and $(1,2,0)$, or one of the two other possibilities obtained from (ii) by permuting the three coordinates.

b. $A=\{(1,1,2),(1,2,1),(2,1,1)\}$, and $B=\{(0,1,2),(0,2,1),(1,0,2)$, $(1,2,0),(2,0,1),(2,1,0),(1,1,1)\}$.

A multifan $(A, B)$ in $Z_{3}^{3}$ cannot have $|A| \geq 4$; otherwise, the condition that $A$ intersects any line segment in at most one point will be violated.

\subsection{WHEN IS $u_{A, B}$ EXTREME IN $Z_{n}^{3}$ ?}

In parallel to Theorem 2.9, Lemma 3.1 and Theorem 3.2, and Theorem 4.5, in this section, we show that $u_{A, B}$ is supermodular and moreover extreme if $(A, B)$ is irreducible.

Given any multifan $(A, B)$, we can find irreducible multifans $\left(A_{i}, B_{i}\right)$ such that

$$
u_{A, B}=\sum_{i} u_{A_{i}, B_{i}}
$$

as follows: Define a relation $R$ on $A$ by $a R a^{\prime}$ if there exists $i, j=1,2$, or 3 such that $p_{i}(a)=p_{j}\left(a^{\prime}\right)$. Let $R^{*}$ be the transitive closure of $R$. Given any equivalence class $A_{1}$, let $B_{1}=\bigcup_{i=1}^{3} p_{i}\left(A_{1}\right)$. Then, $\left(A_{1}, B_{1}\right)$ is a multifan. Suppose we had partitions so that $\left(A_{1}^{\prime}, B_{1}^{\prime}\right)$ and $\left(A_{1}^{\prime \prime}, B_{1}^{\prime \prime}\right)$ were multifans. Then, we cannot have $a^{\prime} \in A_{1}^{\prime}$ and $a^{\prime \prime} \in A_{1}^{\prime \prime}$ such that $a^{\prime} R a^{\prime \prime}$, for this would contradict the fact that $B_{1}^{\prime}$ and $B_{1}^{\prime \prime}$ were disjoint. By an obvious induction, we could not have $a^{\prime}$ in $A_{1}$ and $a^{\prime \prime}$ in $A_{1}^{\prime \prime}$ such that $a^{\prime}$ and $a^{\prime \prime}$ were equivalent under $R^{*}$. This shows that $\left(A_{1}, B_{1}\right)$ is irreducible.

Suppose now that $A_{1}$ and $A_{2}$ are two distinct equivalence classes. We must have that the corresponding $B_{1}$ and $B_{2}$ are disjoint, for if $b$ were a point in both, then $b=p_{i}\left(a_{1}\right)=p_{j}\left(a_{2}\right)$ for some $a_{1} \in A_{1}, a_{2} \in A_{2}$, contradicting the fact that $a_{1}$ and $a_{2}$ are not equivalent. Formula (6.1) follows as the index runs through the various equivalence classes.

THEOREM 6.2. For any multifan $(A, B)$, we have $u_{A, B} \in \mathcal{S}$. In addition, if $(A, B)$ is irreducible, then $u_{A, B}$ is extreme.

Proof. To show supermodularity of $u_{A, B}$, we will show that for any incomparable $(x, y)$, there is an injective function $q: A \cap K_{x, y} \rightarrow B \cap K_{x, y}$. By invoking Theorem 4.8, this shows that $\delta_{u_{A, B}} \geq 0$, since any point in $K_{x, y}$ on which $\hat{u}_{A, B}$ takes a value of -1 will be cancelled out by a value of 1 .

Given any $x, y$, we can suppose, interchanging and reindexing them if necessary, that $x_{1} \geq y_{1}$ and $x_{2} \geq y_{2}$. Take any $a$ in $K_{x, y}$. Then, since $a \not \leq y$, we 
must have at least one index $i$ for which $a_{i}>y_{i}$. If 1 is the only such value of $i$, we define $q(a)=p_{2}(a)$. If there is such a index different than 1 , then we define $q(a)=p_{1}(a)$. In any event, we maintain at least one index $i$ for which $[q(a)]_{i}>y_{i}$, so that $q(a) \leq \leq y$. It is clear that $q(a) \leq a \leq x \vee y$. Now, for $i=1,2$, we have $a_{i} \leq(x \vee y)_{i}=x_{i}$. Since $a \not \leq x$, we must have that $a_{3}>x_{3}$ and, therefore, $[q(a)]_{3}=a_{3}>x_{3}$. Thus, we do not have $q(a) \leq x$, and it follows that $q(a) \in K_{x, y}$.

So, $q(a) \in B \cap K_{x, y}$, and it remains to show that $q$ is injective. Point (i) in the definition of a multifan shows that each of $p_{1}$ and $p_{2}$ is injective, so we need only consider the case for which $q(a)=p_{1}(a)$ and $q\left(a^{\prime}\right)=p_{2}\left(a^{\prime}\right)$ and show that we cannot have $q(a)=q\left(a^{\prime}\right)$. By the definition of $q$, we have $[q(a)]_{j}=a_{j}>y_{j}$ for at least one of $j=2$ or 3 . On the other hand, $\left[q\left(a^{\prime}\right)\right]_{j} \leq a_{j}^{\prime} \leq y_{j}$ for both $j=2$ and 3 . Thus, $q(a) \neq q\left(a^{\prime}\right)$.

Now, suppose that $(A, B)$ is irreducible. We derive the extremeness of $u_{A, B}$ by an argument similar to that of Theorem 4.5, except now it is convenient to use the upper transform. Suppose that $f \preceq u_{A, B}$ with $f \in \mathcal{S}$. We will show that for some nonnegative scalar $\alpha, \hat{f}=\alpha \hat{u}_{A, B}$. First note that from Lemma 4.9, $\hat{f}(x)=$ 0 for all $x \notin A \cup B$, as is true for $\hat{u}_{A, B}$.

For any $a \in A$, define $\alpha(a)$ by $\hat{f}(a)=-\alpha(a)$. It is clear that $\alpha(a) \geq 0$. From Theorem 4.8, $\delta_{u_{A, B}}\left(p_{1}(a), p_{2}(a)\right)=\sum_{z \in K_{p_{1}(a), p_{2}(a)}} \hat{u}_{A, B}(z)=\hat{u}_{A, B}(a)+\hat{u}_{A, B}\left(p_{3}(a)\right)=$ $-1+1=0$. Thus, $\left(p_{1}(a), p_{2}(a)\right)$ is $u_{A, B}$-modular and, thereby, $f$-modular. It follows that $0=\delta_{f}\left(p_{1}(a), p_{2}(a)\right)=\hat{f}(a)+\hat{f}\left(p_{3}(a)\right)$; thus, $\hat{f}\left(p_{3}(a)\right)=\alpha(a)$. Because there was nothing special about the index 3 , we have $f\left(p_{i}(a)\right)=\alpha(a)$ for $i=1,2,3$.

We complete the proof by showing that $\alpha(a)$ is the same for all $a \in A$. It is clearly sufficient to show that $\alpha(a)=\alpha\left(a^{\prime}\right)$ for $a R a^{\prime}$. In this case, we have that for some $i, j=1,2$ or $3, p_{i}(a)=p_{j}\left(a^{\prime}\right)$, so $\alpha(a)=\hat{f}\left(p_{i}(a)\right)=\hat{f}\left(p_{j}\left(a^{\prime}\right)\right)=$ $\alpha\left(a^{\prime}\right)$.

\subsection{EXTREME SUPERMODULAR FUNCTIONS ON $Z_{3}^{3}$}

In this subsection, we determine a complete set of extreme supermodular functions on $Z_{3}^{3}$.

LEMMA 6.3. For all $f \in \mathcal{S}, x \in\left(Z_{n}^{3}\right)^{*}$, and index $i=1,2$, or 3 , we have

$$
\sum_{y \in C(i, x)} \hat{f}(y) \geq 0
$$

Proof. Let $x=(a, b, c)$ and take $i=1$. If either of $b$ or $c=0$, then (6.2) is clear from (4.3). Otherwise, let $x_{1}=(a, b-1, c)$ and $x_{2}=(a, b, c-1)$. Then, $K_{x_{1}, x_{2}}=C(1, x)$, and we can apply Theorem 4.8 to get (6.2). 
THEOREM 6.4. For the lattice $Z_{3}^{3}$, a complete set of extreme supermodular functions is given by the collection of $u_{A, B}$, for $(A, B)$ an irreducible multifan, as listed in Example 6.1.

Proof. We follow the basic strategy described at the end of Section 4.3 with $\mathcal{K}$ equal to the set of all $f \in \mathcal{F}$ such that the sum of all values of $\hat{f}$ over any line segment is nonnegative. By Lemma 6.3, $\mathcal{K} \supseteq \mathcal{S}$.

Given $f \in \mathcal{K}-\mathcal{G}$, define two increasing sequences of sets as follows: Let $A_{1}=\{a\}$, where $a$ is any point on which $\hat{f}<0$. Some such point exists because $f \notin \mathcal{G}$. For each index $i$, choose a point $b_{i}$ on $C(i, a)$ such that $\hat{f}\left(b_{i}\right)>0$. These points must exist by our hypothesis that $f \in \mathcal{K}$. We continue inductively. Suppose $B_{k}$ is defined. For each $b \in B_{k}$ and index $i=1,2,3$, we check if there is a point $a \in A_{k}$ such that $\hat{f}(a)<0$ and $b \in C(i, a)$. If not, we check if there is such a point $a \notin A_{k}$, and if so, then we add that point to $A_{k}$. This leads to a larger set $A_{k+1}$. For each $a \in A_{k+1}$ and index $i=1,2,3$, we check if there is a point $b \in B_{k} \cap C(i, a)$ such that $\hat{f}(b)>0$. If not, there must exist such a point $b \notin B_{k}$, which we add to $B_{k}$. This leads to a larger set $B_{k+1}$. Eventually, we reach an index $n$ such that $A_{n+1}=A_{n}$.

If we can show that neither $A_{n}$ or $B_{n}$ has two points that lie on the same line segment, then it is clear that $\left(A_{n}, B_{n}\right)$ is the multifan we want. Specifically, if $\alpha \leq|\hat{f}(c)|$, where $c \in A_{n} \cup B_{n}$, then $f-\alpha u_{A_{n}, B_{n}} \in \mathcal{K}$ since every line segment containing a point $b \in B_{n}$ will also contain a point $a \in A_{n}$ by the construction.

Suppose, for example, that $A_{n}$ contains distinct points $a$ and $a^{\prime}$ on the same line segment. It follows from the definitions of $A_{n}$ and $B_{n}$ that we would then have a sequence $a=a_{1}, b_{1}, a_{2}, b_{2}, \ldots, a_{k}=a^{\prime}$ and a sequence of indices $i_{1}, i_{2}, \ldots, i_{k}$, where the same index does not appear twice in a row, such that $b_{j} \in C\left(i_{j}, a_{j}\right) \cap C\left(i_{j+1}, a_{j+1}\right)$, and $\hat{f}\left(a_{i}\right)<0$ for all $i=1,2,3$. No element with a zero entry can appear as an $a_{j}$ because, for example, $\hat{f}(x, y, 0) \geq 0$ by (4.3) if $x, y>0$. Also, no element with a zero entry can appear as a $b_{j}$. Otherwise, if say $a_{j}=(x, y, z)$ and $b_{j}=(x, y, 0)$, then $a_{j+1}$ will have its third coordinate equal to zero because $a_{j+1}$ does not line on the same line segment with $b_{j}$ as does $a_{j}$ by construction. Therefore, all the $a_{j}$ and $b_{j}$ in this sequence have coordinates either 1 or 2 . In fact, to go from $a_{j}$ to $b_{j}$, we decrease one coordinate in $a_{j}$ by 1 , then to go from $b_{j}$ to $a_{j+1}$, we increase a different coordinate by 1 . It follows that the sums of the coordinates of any two $a_{j}$ 's are equal. Therefore, $a$ and $a^{\prime}$ cannot be distinct and lie on the same line segment because for this to occur, $a$ and $a^{\prime}$ would differ by 1 in one coordinate.

From the proof, we immediately obtain that to check a function on $Z_{3}^{3}$ for supermodularity, we need only check that the sum of the values of $\hat{f}$ on all line segments is nonnegative.

For $n>3$, the situation is not clear. Now, we can find sequences described as in the proof of Theorem 6.2 for which jumps of two or more in an index are allowed, and we cannot easily rule out the fact that $A_{n}$ or $B_{n}$ contains two 
elements on the same line segment. For example, even in $Z_{4}^{3}$, we have the sequence

$$
(3,2,1),(2,2,1),(2,3,1),(1,3,1),(1,3,3),(1,2,3),(3,2,3)
$$

leading from $(3,2,1)$ to $(3,2,3)$. Some relaxation in the proof is possible. For example, in forming the sets $A_{k}$, we can change the condition on $a$ from being $\hat{f}(a)<0$ to $\hat{f}(a)+\hat{f}(b) \leq 0$. It might well be, however, that we need to adopt a cone smaller than the given one for $\mathcal{K}$.

\section{Summary}

In this paper, we examined the convex cone of supermodular functions on a finite lattice. We were motivated to consider the cone of supermodular functions because it generates the supermodular ordering between random variables, in the sense of Marshall [5]. In Sections 1 and 2, we argued that a base for the modular functions and the extreme rays of the cone of supermodular functions (modulo the modular ones) give one a collection of test functions for determining whether two random variables are ordered according to the supermodular ordering.

Throughout the remainder of the paper, we focused on finding the extreme supermodular functions on various lattices. In Section 3, we completely determined the extreme functions on chain lattices. In Section 4 through 6, we concentrated finding extreme functions on the lattice $Z_{n}^{k}$ and determined a complete set of extreme functions for the lattices $Z_{2}^{3}, Z_{2}^{4}$, and $Z_{3}^{3}$. We conjectured what the extreme functions constitute for the lattice $Z_{2}^{k}$, and we encourage the interested reader to pursue this research.

\section{Acknowledgments}

We thank Alexander Barvinok, participants in the Applied Algebra seminar of York University, and two anonymous referees for helpful comments.

\section{References}

1. Barvinok, A.: A Course in Convexity, Graduate Studies in Mathematics, 54, American Mathematical Society, Providence, Rhode Island, 2002.

2. Edmonds, J.: Submodular functions, matroids, and certain polyhedra, in Guy et al. (eds), Combinatorial Structures and Their Applications; Proceedings, Gordon and Breach, New York, 1970.

3. Kovalëv, M. M.: The cone of symmetric submodular functions, Kibernetika (Kiev) $\mathbf{5}$ (iv) (1985), 122-123. 
4. Kovalëv, M. M., Zaporozhets, A. A. and Girlikh E.: Characterization of some subclasses of Monge matrices, Dokl. Nac. Akad. Nauk Belarusi 42 (1998), 38-44.

5. Marshall, A. W.: Multivariate stochastic orderings and generating cones of functions, in K. Mosler and M. Scarsini (eds.), Stochastic Orders and Decision under Risk, IMS Lecture Notes - Monograph Series, 19, 1991, pp. 231-247.

6. Mosler, K. and Scarsini, M.: Stochastic Orders and Applications: A Classified Bibliography, Lecture Notes in Economics and Mathematical Systems, 401, Springer-Verlag, Berlin, 1993.

7. Müller, A.: Stochastic orders generated by integrals: A unified study, Adv. Appl. Probab. 29 (1997), 414-428.

8. Müller, A. and Scarsini, M.: Some remarks on the supermodular order, J. Multivar. Anal. 73 (2000), 107-119.

9. Müller, A. and Stoyan, D.: Comparison Methods for Stochastic Models and Risks, John Wiley \& Sons, New York, 2002.

10. Promislow, S. D.: Stochastic orders on discrete multivariate distributions, working paper, Department of Mathematics and Statistics, York University, 2004.

11. Shaked, M. and Shanthikumar, J. G.: Supermodular stochastic orders and positive dependence of random variables, J. Multiv. Anal. 61 (1997), 86-101.

12. Topkis, D. M.: Minimizing a submodular function on a lattice, Oper. Res. 26 (1978), 305321.

13. Whitt, W.: Stochastic comparisons for non-Markov processes, Math. Oper. Res. 11 (4) (1986), 608-617.

14. Ziegler, G. M.: Lectures on Polytopes, Graduate Texts in Mathematics, Springer-Verlag, New York, 1995. 